Schweiz. Z. Tuberk. 1956;13:I-IV

\title{
Contents. Vol. 13, 1956
}

\section{INDEX}

Aguet, F., et G. Гavez

Aguet, F. Arnold, E.

Baudet, P. Birkhäuser, H.

Blöchlinger-Koller, A.

Bohn, W., und W. Singer

Bossy, A. Brisk, D.T. Brunner, W.

Cevey, M. Chauvet, M. Depraz, A. Dettwiler, E. Düggeli, O. Van Erpecum, C. P.

Favez, G., F. Aguet et A. Bossy

Favez, G.

Forster, E.

Garbinskí, T., und F. Ludewig

Gilbert, M., et A. Depraz

Giovanoli, P.F.

Hartwig, H., G. Heuer und H. Neubert

Essai d'interprétation des faits cliniques an moyen des résultats expérimentaux dans le traitement de la tuberculose par les hormones cortico-snrrénaliennes 498

vide Favez, G.

Les examens de la fonction respiratoire

vide Koch, J.

vide Koch J.

Über die Beziehungen zwischen Tuberkulose und

Psyche

395

Erste Erfahrungen mit BCG-Impfungen im Kanton

Basel-Stadt 256

Die Bronchographie mit Propyliodon-Cilag-Suspen

sion, unter besonderer Berücksichtigung der Be-

schlagdarstellung $\quad 81$

vide Favez, G.

vide Koch, J.

Erfahrungen mit der doppelseitigen Resektion zur

Behandlung der Lungentuberkulose 515

vide Michetti, D.

La biopsie de Daniels en pneumologie

vide Gilbert, $\mathrm{M}$.

vide Meyer, $\mathrm{H}$.

Klinische Erfahrungen mit Pyrazinamid 
Über die unspezifíschen, bakteriellen Infektionen bei Lungentuberkulose und ihre Beziehungen zum eosinophilen Bronchialkatarrh 459

Le traitement de la tuberculose pulmonaire evolutive par les perfusions courtes de PAS quotidiennes et biquotidiennes 1

vide Aguet, F.

vide Vaucher, E.

Sonnenbestrahlungen bei Lungentuberkulose unter Antistin-Schutz 440

Apparition, remaniements, regression d'images bulleuses au cours d'une tuberculose pulmonaire

Pneumoperitoneum und temporäre Phrenicusaus-schaltung zur Behandlung der Lungentuberkulose 210

Das Verhalten des Bluteiweißbildes bei der intravenösen Behandlung der Lungentuberkulose mit Para-Aminosalicylsäure (PAS) 89

Heuer, G.

HOFSTETTER, M.

Houriet, J.H.

Inama, K., und K. Larcher

Jentgens, H. Kartagener, M., und

K. MÜLLY

Kartagener, M., und K. Mülly

Koch, J., P. Baudet und D.T. Brisk

Koch, J.

Larcher, K.

Lauener, $\mathrm{H}$.

Lemoine, J.M.

Lotz, H.H., und H.W. Rautenburg

Ludewig, F.

Mähki, H.H., und E. Tanner

Michetti, D., et M. Cevey

Meyer, H., H. Lauener und E. Dettwiler

Mülly, K. Naef, A. P.

Nagy el Mehallawy, M.

Neubert, $\mathrm{H}$.

Nick, J.

Pozsonyi, J., und V. Wohl

Rautenburg, H.W.

Roegel, E.

Salib, M., M. Nagy el Mehallawy und S. Shash

Shash, S.

Singer, W.

vide Iíartwig, $\mathrm{H}$. 
Die Behandlung der Lungentuberkulose mit dem Hydrazid der Cyanessigsäure (CEH) (Reazide) .... 430

Presentation de cas 464

Pathologisch-anatomische und klinische Betrachtun.

gen zur Streptomycinbehandlung der Tuberkulose . 110

Tuberkulose und Schwangerschaft 41

Familiares Vorkommen von Bronchiektasien

Bronchiektasien bei Situs yiscerum invcrsus 166

Vergleichende Bestimmungen der Serumkonzentra-

tionen Cyanessigsäurehydracid (CEH) und Isonicotin-

säurehydracid (INH) 139

Zur Differentialdiagnose, Prognose und Therapie der

tuberkulösen Rundherdformen der Lunge 407

vide Inama, $\mathrm{K}$.

vide Meyer, $\mathrm{H}$.

La place de la tuberculose bronchique en phtisiologie 278

Über die Pathogenese der Leptomeningitis tuber-

culosa 263

vide Garbiáski, T.

Oxamycin (Cycloserin)

483

A la recherche du potentiel évolutif des foyers tuberculeux 543

Blutspiegel- und Ausscheidungsverhältnisse bei der

Benzacyl-Therapie mit Beriicksichtigung der Kumu-

Iationsfrage $\quad 420$

vide Kartagener, M.

Le problème de l'étendue topographique de l'exérèse

en tuberculose pulmonaire 521

vide Salib, M.

vide Hartwig, $\mathrm{H}$.

Weitere Beobachtungen mit Pyrazinamid 490

Liquor-Untersuchungen bei tuberkulöser Spondylitis

im Kindesalter 201

vide Lotz, H. H.

vide Vaucher, E.

Intrapleurale Applikation von Isoniazid zur Behandlung der primären tuberkulösen Pleuritís

exsudativa 67

vide Salib, M.

vide Bohn, W.

Tannek, E.

Trendelenburg, F.

Vaucher, E., E. Forstek et

E. ROEGEL VISCHER, W.A.

VISCHER, W.A.

VOLUTER, G.

WOHL, V. 
vide Märki, H.H.

Die Vinilenzminderung INH-reßistenter Tuberkel-

bakterien in Experiment und Klinik 528

La place de la tomographie transverso-axiale dans le

diagnostic d'opérabilité des cancers du poumon, ses

possibilités et limites 156

Auswertung eines einfachen Blutagarnährbodens als

Medium znr Resistenzbestimmung von Tuberkel-

bakterien gegen Chemotherapeutica 34

Über die Konstanz der Virulenzabschwächung

INH-resistenter Tuberkelbakterien für das Meer-

schweinchen 537

A propos du phénomène radiologique du «Cceur toxique» dans les primo-infections

tuberculeuses .... 97

vide Pozsonyi, J.

\section{SOCIETATES}

\section{LIBRI}

Nekrologia Varia

Schweiz. Vereinigung gegen die Tuberkulose. Gesell-schaft schweiz. Tuberkuloseärzte. 36. wissenschaft-lichc Tagung in Neuenburg, 28./29. April 1956 - Association Suisse centre la Tuberculose. Société des Médecins Suisses specialises en Tuberculose, 36e reunion scientifique à Neuchâtel, les 28/29 avril 1956453

$74,147,219,314,448,556$

Morin, J 153

Rist, E 313

Maurer, G 317

78,452

Alle Rechte, insbesoudere das der Übersetzung in fremde Sprachen, vorbebalten.

Ohne ausdrückliche Genehmigung des Verlages ist es auch nicht gestattet, diesen Band oder

Teile

daraus auf photomechanischem Wege (Photokopîe, Mikrokopie) zu vervielfältigen.

(C)

Printed in Switzerland

Copyright 1956 by S. Karger AG., Basel

by Basler Druck- und Verlagsanstalt, Basel

Cliches: Aberegg-Steiner \& Cie., AG., Bern, und Steiner \& Cic, AG., Basel 